\title{
Review
}

\section{Eggs to die for: cell death during Drosophila oogenesis}

\author{
M Buszczak ${ }^{1}$ and L Cooley ${ }^{\star 2,3}$ \\ 1 Department of Molecular, Cellular and Developmental Biology, Yale University, \\ New Haven, CT 06520-8103, USA \\ 2 Department of Genetics, Yale School of Medicine, 333 Cedar Street, New \\ Haven, CT 06510, USA \\ ${ }^{3}$ Department of Cell Biology, Yale School of Medicine, 333 Cedar Street, New \\ Haven, CT 06510, USA \\ * Corresponding author: L Cooley, Department of Genetics, Yale School of \\ Medicine, 333 Cedar Street, New Haven, CT 06510, USA. Tel: 203-785-5067; \\ Fax: 203-785-6333; E-mail: lynn.cooley@yale.edu
}

Received 10.7.00; revised 3.8.00; accepted 7.8.00

Edited by S Kumar

\begin{abstract}
Extensive programmed cell death occurs in the female germline of many species ranging from $C$. elegans to humans. One purpose for germline apoptosis is to remove defective cells unable to develop into fertile eggs. In addition, recent work suggests that the death of specific germline cells may also play a vital role by providing essential nutrients to the surviving oocytes. In Drosophila, the genetic control of germline apoptosis and the proteins that carry out the death sentences are beginning to emerge from studies of female sterile mutations. These studies suggest that the morphological changes that occur during the late stages of Drosophila oogenesis may be initiated and driven by a modified form of programmed cell death. Cell Death and Differentiation (2000) 7 , 1071-1074.
\end{abstract}

Keywords: apoptosis; germline; nurse cell; Drosophila

The process of programmed cell death, or apoptosis, eliminates unwanted or unneeded cells in proliferating and developing tissues. The occurrence of programmed cell death is a common feature of female germline development in many species including nematodes, insects and mammals. ${ }^{1-5}$ In $C$. elegans, a systematic analysis has shown that a reproducible number of syncytial germ cells undergo programmed cell death. ${ }^{5}$ In human fetal development, the peak number of ovarian germline cells is almost seven million at 5 months gestation; this number plummets to two million at birth as the result of massive cell death. ${ }^{6}$ Although the exact function of this extensive germline apoptosis remains unclear, work in $C$. elegans suggests that the destruction of excess cells may provide surviving cells with nutrients essential for the completion of oogenesis. ${ }^{5}$ In Drosophila, this is most certainly the case, where the development of each mature egg is always accompanied by the apoptosis of its 15 sister nurse cells at the end of oogenesis. The predictability of this cell death allows for the genetic analysis and cellular characterization of discrete events leading up to and occurring throughout apoptosis.

Each Drosophila egg develops within a follicle called an egg chamber that contains cells from both germline and somatic lineages. ${ }^{7}$ Somatic follicle cells surround the egg chamber providing the proper environment for the development of the 16 germline cells within. The 15 nurse cells and one oocyte are derived from a single germline precursor cell by four mitotic divisions. As in many other species, cytokinesis during these mitotic cycles is incomplete leaving a cytoplasmic bridge connecting the cells. In Drosophila, the bridges become stabilized by an actin cytoskeleton to become ring canals, through which cytoplasm flows into the oocyte. ${ }^{8}$ Because of the large overall size of egg chambers, the morphological changes that occur during oogenesis are easily distinguished using a light microscope. Egg chambers develop sequentially within tubular ovarioles, so a single ovary contains egg chambers representing all stages of oogenesis.

The discovery that Drosophila egg chambers could undergo programmed cell death was first made nearly 25 years ago. This analysis, using light and electron microscopy, found that some egg chambers degenerate almost exclusively between stages 7 and 8 of oogenesis. ${ }^{9}$ The germline cells of these egg chambers exhibit the morphological hallmarks of apoptosis, including chromatin condensation and membrane blebbing. The follicle cells, which remain remarkably intact while the germline cells die, phagocytize the cellular remnants of the germline after they have completed their apoptotic program. The observation that egg chambers exhibit the greatest frequency of degeneration during stages 7 and 8 suggests that midoogenesis may be the point at which defective egg chambers are eliminated. The availability of food and other environmental cues can also influence the ability of egg chambers to develop beyond stage $8 .^{7}$ Furthermore, several mutations result in egg chamber arrest and degeneration during mid-oogenesis. Interestingly, egg chambers enter vitellogenesis during stage 8 and this process represents a significant investment of energy and resources on the part of the female. Therefore a previtellogenic checkpoint may serve to eliminate egg chambers unable to produce viable progeny and thus prevent the waste of precious nutrients. Recent evidence indicates that the ecdysone response hierarchy ${ }^{10}$ is an important element of this checkpoint. ${ }^{11}$ Egg chambers unable to synthesize or respond to ecdysone undergo apoptosis during mid-oogenesis. How the ecdysone pathway interacts with the cell death machinery remains under investigation (see article by Baehrecke in this issue).

Besides removing defective egg chambers, programmed cell death plays an integral role in the normal development of every oocyte. As their moniker implies, nurse cells 
supply the developing oocyte with nutrients throughout oogenesis. ${ }^{12}$ Transport from the nurse cells to the oocyte occurs in two phases: a slow phase during which specific molecules are passed into the oocyte, and a rapid phase, also referred to as dumping, during which the nurse cells empty all their remaining cytoplasm into the oocyte. At stage $10 \mathrm{~B}$, nurse cells prepare for the rapid phase of transport with a dramatic rearrangement of filamentous actin to form actin bundles. These actin bundles extend from the plasma membrane, radiate towards the nuclear membrane and provide structural support for the large polyploid nuclei (Figure 1B). Next, the nurse cell nuclear envelopes become permeabilized (Figure 1F), followed by a myosin-based contraction of the cortical actin of each nurse cell during stage 11. After the completion of dumping, the depleted nurse cells are cleared from the egg chamber during stages 12 and 13 .

Several recent studies have shown that nurse cells undergo apoptosis. DNA extracted from ovaries contains nucleosome-sized fragments. ${ }^{3,4}$ Acridine orange and TUNEL (Figure 2) further confirm the fragmentation of nurse cell DNA during stages 12 and 13 after the completion of dumping. ${ }^{3,4}$ However, the process of dumping does not appear to cause the death of nurse cells. TUNEL indicates that DNA fragmentation still occurs, albeit slightly delayed, in mutant egg chambers unable to complete rapid cytoplasm transport. ${ }^{3,4}$ These data leave open the questions of what signals induce nurse cell apoptosis and at what stage do the nurse cells receive these signals. Interestingly, electron micrographs reveal that nurse cell nuclei have already begun to exhibit signs of apoptosis during dumping at stage 11; the chromatin and nucleolar material gathers in small round masses and the nuclear envelope becomes ruffled (Figure $1 \mathrm{H}$ ). ${ }^{13}$ Furthermore, levels of free calcium in the nurse cytoplasm increase during stage $11 .{ }^{14}$ Similar fluxes in free calcium are observed in murine cells undergoing apoptosis. ${ }^{15,16}$

Other molecular markers indicate that preliminary symptoms of nurse cell apoptosis can be traced back to stage $10 \mathrm{~B}$, the time when these cells are preparing for fast cytoplasm transport. During embryogenesis, transcripts of the caspase DREDD accumulate in cells specified to enter apoptosis. ${ }^{17}$ During oogenesis, there is a dramatic increase in DREDD expression at stage 10 (Figure 3). ${ }^{17}$ Expression of the ecdysone-inducible caspase DRONC also appears to be upregulated at this time. ${ }^{18}$ Early in stage 10, anti-cytochrome c antibodies fail to stain egg chambers; however, at stage 10B, these antibodies stain nurse cells in a punctuate pattern reminiscent of mitochondria (Figure 3). ${ }^{19}$ Furthermore, the genes for the caspase DECAY and the Bcl-2 homolog DEBCL are expressed throughout oogenesis. ${ }^{20,21}$ While expression does not imply function, these data show that core components of the cell death pathway are present during oogenesis. The increased expression of certain caspases and the increased immunoreactivity of anti-cytochrome $c$ antibodies suggest that although the nurse cells do not display any overt signs of apoptosis at stage 10B, the events that lead to their ultimate demise have already been set in motion.

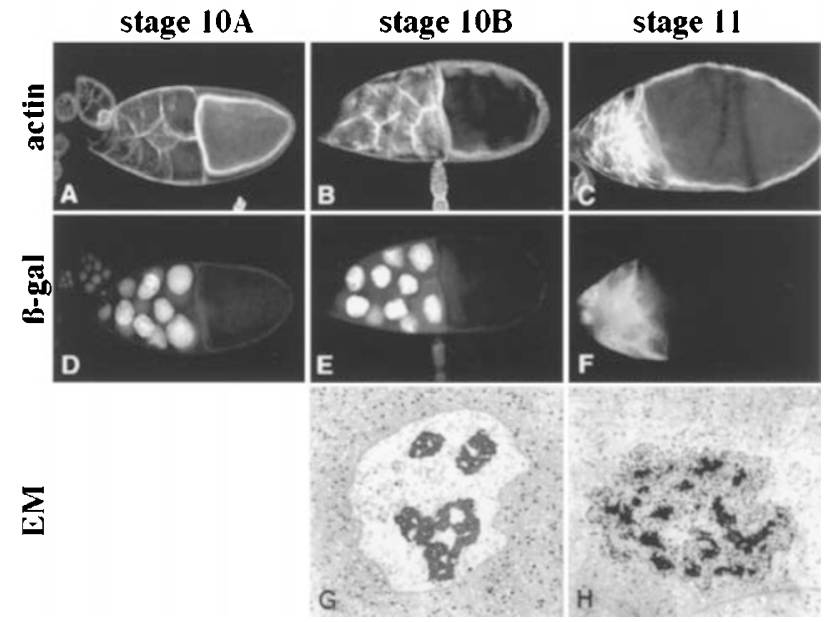

Figure 1 Morphological changes associated with rapid cytoplasm transport. Egg chambers were stained with rhodamine-conjugated phalloidin $(\mathbf{A}-\mathbf{C})$ to visualize the actin cytoskeleton or with anti- $\beta$-galactosidase ( $\beta$-gal) antibody (D-F) to detect the expression of nuclear $\beta$-gal from a germline enhancer trap. (A) Until stage 10A filamentous actin (F-actin) localizes to ring canals and to the cortex of nurse cells and follicle cells. (B) At stage 10B, there is a dramatic rearrangement of $\mathrm{F}$-actin to form actin bundles that extend from the nurse cell plasma membrane to the nuclei. (D, E) During stages $10 \mathrm{~A}$ and $10 \mathrm{~B}, \beta$-gal remains tightly contained within the nurse cell nuclei. (F) After stage 10B and into stage 11 , the nurse cell nuclei become perforated allowing $\beta$-gal to leak into the cytoplasm. (C, F) During stage 11, the nurse cells contract and force their cytoplasm into the oocyte. $(\mathbf{G}, \mathbf{H})$ Differences in nurse cell nuclear morphology can be detected between stages $10(\mathbf{G})$ and $11(\mathbf{H})$ by electron microscopy (EM). By stage 11, chromatin condensation and nuclear membrane ruffling are observed (electron micrographs courtesy of Shaw and Tilney ${ }^{13}$ )
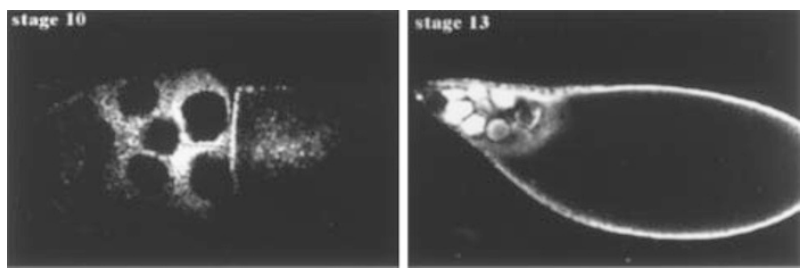

Figure 2 TUNEL in egg chambers. During stage 10, there is no apparent DNA degradation in nurse cells. After the completion of dumping, extensive TUNEL is observed in nurse cells (stage 13 shown) (TUNEL images courtesy of Foley ${ }^{4}$ )

Recent genetic analysis has suggested that programmed cell death may actually trigger the rapid phase cytoplasm transport. Germline clones of $d c p-1$ mutations were made in an attempt to remove the maternal component of this caspase from embryos. However, females carrying $d c p-1$ homozyous clones were sterile and produced small, inviable eggs. ${ }^{22}$ At stage $10 \mathrm{~B}, d c p-1$ germline clonal egg chambers displayed numerous defects. Staining mutant egg chambers with rhodamine-conjugated phalloidin showed that loss of $d c p-1$ caused a delay in actin bundle formation. ${ }^{22}$ Moreover, analysis using anti-lamin antibodies and a germline enhancer trap revealed that $d c p-1$ clonal egg chambers exhibited defects in nurse cell nuclear envelope perforation and contraction. ${ }^{22}$ These defects led to an overall dumpless phenotype and showed that this 


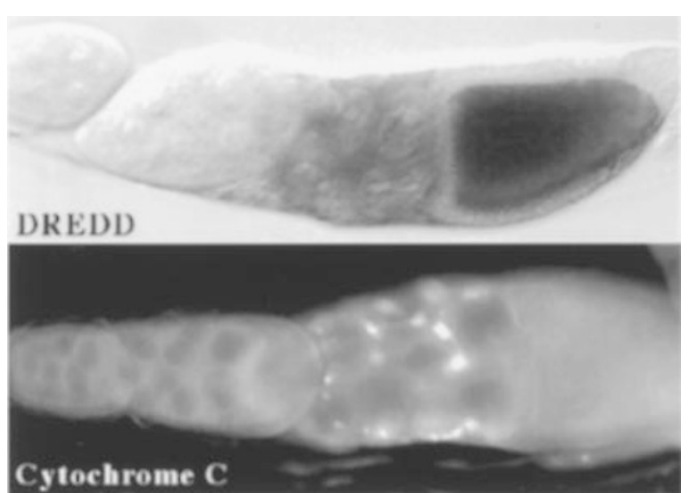

Figure 3 Detection of apoptotic markers at stage 10 of oogenesis. Egg chambers were stained with dredd mRNA probes or anti-cytochrome $c$ antibodies. The amount of detectable dredd mRNA dramatically increases at stage 10. Similarly, cytochrome $c$ immunoreactivity is first observed in stage 10 nurse cells (dredd mRNA in situ image courtesy of Rodriguez and Abrams $^{17}$; cytochrome-c image courtesy of Varkey and Abrams ${ }^{19}$ )

caspase is required to initiate fast cytoplasm transport and the suicide of nurse cells.

Previous work demonstrated that actin bundle formation and nurse cell contraction are regulated by separate genetic pathways (Figure 4). For instance, mutations in chickadee (chic), singed (sn) and quail (qua) result in female sterility due to defects in actin bundle formation. ${ }^{23-}$ 25 In the absence of actin bundles, nurse cell nuclear permeabilization and the ability of the nurse cells to contract are not affected. When contraction occurs, however, the nuclei move and occlude ring canals, thereby preventing the further flow of cytoplasm into the oocyte. In contrast, loss of the myosin regulatory light chain, encoded by the spaghetti squash (sqh) gene disrupts nurse cell contraction but has no effect on actin bundle formation. ${ }^{26,27}$ These data demonstrate that independent but parallel genetic pathways control actin bundle formation and nurse cell contraction. Germline clonal analysis using mutations in $d c p-1$ suggest that entry into apoptosis is required to carry out both of these pathways, which culminate in rapid cytoplasm transport and the elimination of the nurse cells (Figure 4).

The search for other effectors of nurse cell death has revealed surprising differences compared to somatic cell death. Initial studies of embryonic programmed cell death in Drosophila led to the identification of three positive effectors of apoptosis. These three genes, head involution defective (hid), grim and reaper (rpr), are tightly linked and uncovered by a small deletion $\mathrm{Df}(3 \mathrm{~L}) \mathrm{H} 99^{28-30}$ Expression of $r p r$ and hid in the nurse cells begins at stage $9 .{ }^{4}$ To test whether the expression of these genes induced nurse cell apoptosis, germline clones of the Df(3)H99 deficiency were generated. Surprisingly, loss of hid, grim and $r p r$ did not disrupt the rapid phase of transport. ${ }^{4}$ Nurse cells from these clonal egg chambers were cleared on time and the resulting eggs were wild-type in size. Therefore hid, grim and $r p r$ are not required in the germline.

Although the proteins that directly induce caspase activation in the germline remain unknown, more than one

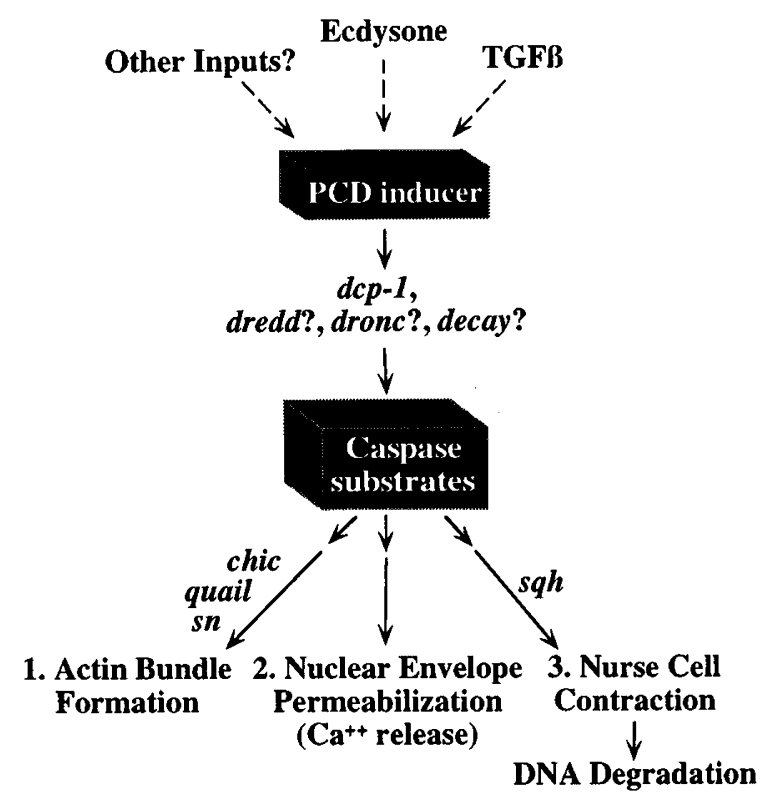

Figure 4 A model for apoptosis driving rapid cytoplasm transport. Available evidence suggests that several signaling pathways may regulate the expression or activity of an unidentified inducer of apoptosis. This inducer activates DCP-1 and possibly other caspases including DREDD, DRONC and DECAY. Activated caspases cleave unknown downstream substrates, which in turn, trigger parallel genetic pathways leading to rapid cytoplasm transport

signaling pathway may contribute to the regulation of this process. For example, the steroid hormone ecdysone regulates programmed cell death during metamorpho$\operatorname{sis}^{31,32}$ and there is some evidence that ecdysone can influence cell death in the ovary. Injecting females with ecdysone results in the premature apoptosis of stage 9 egg chambers. ${ }^{33}$ Therefore, ecdysone may be needed not only as a permissive cue to initiate vitellogenesis, ${ }^{11}$ but also as a pro-apoptotic signal in nurse cells.

Conditional mutations in the $d p p$ gene, which encodes a TGF $\beta$ family member, result in the production of viable adults at the permissive temperature. ${ }^{34}$ When these adults are shifted to the restrictive temperature, females often produce small eggs. Furthermore, disruption of the TGF $\beta$ receptor gene saxaphone (sax) causes defects in both actin bundle formation and the rapid phase of cytoplasm transport. ${ }^{34}$ Further analysis is needed to clarify whether mutants of the $d p p$ signaling pathway affect all the morphological changes in the nurse cells involved with the process of dumping. Intriguingly, $d p p$ is expressed in the follicle cells that overlie the nurse cells, and the DPP receptor genes are expressed in the nurse cells. ${ }^{34}$ Therefore, these results suggest the exciting possibility that a signal from the follicle cells contributes to inducing programmed cell death in the germline.

The genetically tractable fruit fly has proven to be an invaluable model system for studying programmed cell death. Studies in Drosophila and C. elegans show that many germline cells are sacrificed for the benefit of surviving oocytes. Perhaps these findings reflect a function for the widespread incidence of germ cell death in many evolutionarily diverse organisms. While recent work 
suggests that different inducers of apoptosis operate in Drosophila germline and somatic cells, the molecules that execute the cell death program are likely to be the same. Interestingly, a similar observation has been made in $C$. elegans where the novel $\mathrm{BH} 3$ domain-containing protein EGL-1, which is required for somatic cell death, does not function during apoptosis in the germline. ${ }^{5}$ Many important questions remain to be answered about Drosophila germline apoptosis. What is the identity of the germline apoptosis inducer? What protects the oocyte from the same fate as its sister nurse cells? What are the downstream targets of caspases and how does their cleavage result in the morphological changes in nurse cells? The genetic and cell biological advantages inherent to the study of Drosophila oogenesis will yield new insights into the mechanisms driving germline apoptosis.

\section{Acknowledgements}

We would like to thank M Shaw, L Tilney, K Foley, A Rodriguez, J Varkey and $\mathrm{J}$ Abrams for images used in this review. This work was supported by a grant from the NIH to L Cooley (GM43301).

\section{References}

1. De Pol A, Vaccina F, Forabosco A, Cavazzuti E and Marzona L (1997) Apoptosis of germ cells during human prenatal oogenesis. Hum. Reprod. 12: 2235-2241

2. De Pol A, Marzona L, Vaccina F, Negro R, Sena $P$ and Forabosco A (1998) Apoptosis in different stages of human oogenesis. Anticancer Res. 18: $3457-$ 3461

3. Cavaliere V, Taddei $C$ and Gargiulo G (1998) Apoptosis of nurse cells at the late stages of oogenesis of Drosophila melanogaster. Dev. Genes Evol. 208: 106 112

4. Foley K and Cooley L (1998) Apoptosis in late stage Drosophila nurse cells does not require genes within the H99 deficiency. Development 125: 1075-1082

5. Gumienny TL, Lambie E, Hartwieg E, Horvitz HR and Hengartner MO (1999) Genetic control of programmed cell death in the Caenorhabditis elegans hermaphrodite germline. Development 126: 1011-1022

6. Baker TG and Sum W (1976) Development of the ovary and oogenesis. Clin. Obstet. Gynaecol. 3: 3-26

7. Spradling AC (1993) Developmental Genetics of Oogenesis. In: Bate M, Martinez Arias A (eds). The Development of Drosophila menalogaster. Cold Spring Harbor Laboratory Press: Cold Spring Harbor, pp. 1-70

8. Robinson DN and Cooley L (1996) Stable intercellular bridges in development: the cytoskeleton lining the tunnel. Trends Cell Biol. 6: 474-479

9. Giorgi F and Deri $P$ (1976) Cell death in ovarian chambers of Drosophila melanogaster. J. Embryol. Exp. Morph. 35: 521-533

10. Thummel CS (1996) Flies on steroids-Drosophila metamorphosis and the mechanisms of steroid hormone action. Trends Genet. 12: 306-310

11. Buszczak M, Freeman MR, Carlson JR, Bender M, Cooley L and Segraves WA (1999) Ecdysone response genes govern egg chamber development during midoogenesis in Drosophila. Development 126: 4581-4589

12. Mahajan-Miklos S and Cooley L (1994) Intercellular cytoplasm transport during Drosophila oogenesis. Dev. Biol. 165: 336-351
13. Guild GM, Connelly PS, Shaw MK and Tilney LG (1997) Actin filament cables in Drosophila nurse cells are composed of modules that slide passively past one another during dumping. J. Cell Biol. 138: 783-797

14. Matova N, Mahajaran-Miklos S, Mooseker MS and Cooley L (1999) Drosophila quail, a villin-related protein, bundles actin filaments in apoptotic nurse cells. Development 126: 5645-5657

15. Lam M, Dubyak G and Distelhorst CW (1993) Effect of glucocorticosteroid treatment on intracellular calcium homeostasis in mouse lymphoma cells. Mol. Endocrinol. 7: 686-693

16. Lam M, Dubyak G, Chen L, Nunez G, Miesfeld RL and Distelhorst CW (1994) Evidence that $B C L-2$ represses apoptosis by regulating endoplasmic reticulumassociated $\mathrm{Ca}^{2+}$ fluxes. Proc. Natl. Acad. Sci. USA 91: 6569-6573

17. Chen P, Rodriguez A, Erskine R, Thach T and Abrams JM (1998) Dredd, a novel effector of the apoptosis activators reaper, grim, and hid in Drosophila. Dev. Biol. 201: 202-216

18. Dorstyn L, Colussi PA, Quinn LM, Richardson Hand Kumar S (1999) DRONC, an ecdysone-inducible Drosophila caspase. Proc. Natl. Acad. Sci. USA 96: 4307 4312

19. Varkey J, Chen P, Jemmerson R and Abrams JM (1999) Altered cytochrome $c$ display precedes apoptotic cell death in Drosophila. J. Cell Biol. 144: 701-710

20. Dorstyn L, Read SH, Quinn LM, Richardson H and Kumar S (1999) DECAY, a novel Drosophila caspase related to mammalian caspase-3 and caspase-7. J. Biol. Chem. 274: 30778-30783

21. Colussi PA, Quinn LM, Huang DC, Coombe M, Read SH, Richardson $H$ and Kumar S (2000) Debcl, a proapoptotic Bcl-2 homologue, is a component of the Drosophila melanogastercell death machinery. J. Cell Biol. 148: 703-714

22. McCall Kand StellerH (1998) Requirement forDCP-1 caspase during Drosophila oogenesis. Science 279: 230-234

23. Cooley L, Verheyen E and Ayers K (1992) chickadee encodes a profilin required for intercellular cytoplasm transport during Drosophila oogenesis. Cell 69: 173184

24. Cant K, Knowles BA, Mooseker MS and Cooley L (1994) Drosophila singed, a fascin homolog, is required for actin bundle formation during oogenesis and bristle extension. J. Cell Biol. 125: 369-380

25. Mahajan-Miklos S and Cooley L (1994) The villin-like protein encoded by the Drosophila quail gene is required for actin bundle assembly during oogenesis. Cell 78: 291-301

26. Wheatley S, Kulkarni S and Karess R (1995) Drosophila nonmuscle myosin II is required for rapid cytoplasmic transport during oogenesis and for axial nuclear migration in early embryos. Development 121: 1937-1946

27. Edwards KA and KiehartDP (1996) Drosophila nonmuscle myosin II has multiple roles in imaginal discs and egg chamber morphogenesis. Development 122: $1499-1511$

28. White K, Grether ME, Abrams JM, Young L, Farrell Kand Steller H (1994) Genetic control of programmed cell death in Drosophila. Science 264: 677-683

29. Grether ME, Abrams JM, Agapite J, White K and Steller H (1995) The head involution defective gene of Drosophila melanogaster functions in programmed cell death. Genes Dev. 9: 1694-1708

30. Chen P, Nordstrom W, Gish B and Abrams JM (1996) grim, a novel cell death gene in Drosophila. Genes Dev. 10: 1773-1782

31. Jiang C, Baehrecke EH and Thummel CS (1997) Steroid regulated programmed cell death during Drosophila metamorphosis. Development 124: 4673-4683

32. Jiang C, Lamblin AF, Steller H and Thummel CS (2000) A steroid-triggered transcriptional hierarchy controls salivary gland cell death during Drosophila metamorphosis. Mol. Cell 5: 445-455

33. Soller M, Bownes M and Kubli E (1999) Control of oocyte maturation in sexually mature Drosophila females. Dev. Biol. 208: 337-351

34. Twombly V, Blackman RK, Jin H, Graff JM, Padgett RW and Gelbart WM (1996) The TGF-beta signaling pathway is essential for Drosophila oogenesis. Development 122: 1555-1565 